\title{
Immunohistochemical demonstration of carcinoembryonic antigen, amylase, secretory component, immunoglobulin A and lysozyme in human minor salivary gland tumors
}

\section{-A preliminary study-}

\author{
Ichiro Saito, Machiko Miyake, Katsunori Suto and Hidesaburo Kusuhara \\ Department of Pathology (Chief: Prof. Shinichiro Umemura), Nihon University \\ School of Dentistry, 1-8-13, Surugadai Kanda, Chiyoda-ku, Tokyo 101, Japan
}

[Accepted for publication: October 20, 1982]

\begin{abstract}
Key words: immunohistochemistry/carcinoembryonic antigen (CEA)/secretory proteins/minor salivary gland tumors
\end{abstract}

\section{Introduction}

Carcinoembryonic antigen (CEA) was discovered in 1965 by Gold and Freedman ${ }^{11}$. Subsequent immunohistological studies have confirmed that CEA is present not only in colon cancer, carcinomas of the non-digestive organ and salivary gland tumors but even in normal organs as well. Hence, many investigators have been of the opinion that CEA is not specific for cancer but rather has a tendency to increase in amount in the presence of cancer ${ }^{2}$.

Immunoglobulin secreted in the salivary glands plays a major role in the local immune mechanism of the oral field as has been shown by application of immunohistological techniques and immunoelectron microscopy.

CEA and immunoglobulins have often been studies separately but seldom with regard to their correlation.

These considerations led us to undertake an immunohistological study of five secretory proteins, i.e., CEA, amylase, secretory component (SC), immunoglobulin A $(\operatorname{IgA})$, and lysozyme, in order to examine changes in their localization in relation to carcinogenesis and with a view to recognizing the biological properties and functional profiles of tumors in the minor salivary gland.

\section{Materials and Methods}

Twenty-one cases of primary minor salivary gland tumors observed at the Nihon University Dental Hospital were used. Included were 11 cases of pleomorphic adenoma (PMA), 7 mucoepidermoid tumor (MET), and 3 adenoid cystic carcinoma (ACC). Histologicaly normal minor salivary glands attached to the resected tumors served as controls, together with normal colon mucosa and colon cancers obtained from autopsy and biopsy.

For localization of CEA (DAKO-patts) $1: 10$, an immunohistochemical technique using the peroxidase-antiperoxidase (PAP) method of Sternberger et al.3) was applied in $5 \mu \mathrm{m}$ sections of formalin-fixed tissue and paraffin-embedded tissue.

Immunohistochemical examinations of amylase (Nordic) 1:20, SC (DAKO-patts) 1:20, IgA (Behling) 1:20, and lysozyme (DAKO-patts) 1:40 with PAP method in formalin-fixed and paraffin-embedded sections were processed in the same way as described above.

To increase antigenicity, the sections were incubated in $0.3 \%$ trypsin for $15 \mathrm{~min}$ at room temperature before treatment with goat serum ${ }^{4}$. Control of the staining was performed by using normal rabbit serum in place of the specific antiserum or by omiting the antiserum. 


\section{Results and Discussion}

\section{CEA}

The results for all normal minor salivary glands were negative. In most PMA, the luminal border of the ductal cells was weakly positive. In MET, among the 3 categories of well, moderately, and poorly differentiated, as classified by Healey et al. ${ }^{5)}$, the poorly differentiated group had a strong posi- tive. The localization of CEA were small tubules and were situated adjacent to the lumina (Fig. 1). The clear cells at the periphery of the tubules were always negative. Cells forming cyst-like space with a typical cribriform pattern and lumen of duct-like structure had positive ACC findings (Fig. 2). In both ACC and MET with a high degree of positive in the poorly differentiated group, the CEA reaction was proportional to the degree of malignancy as reported by Ejek-

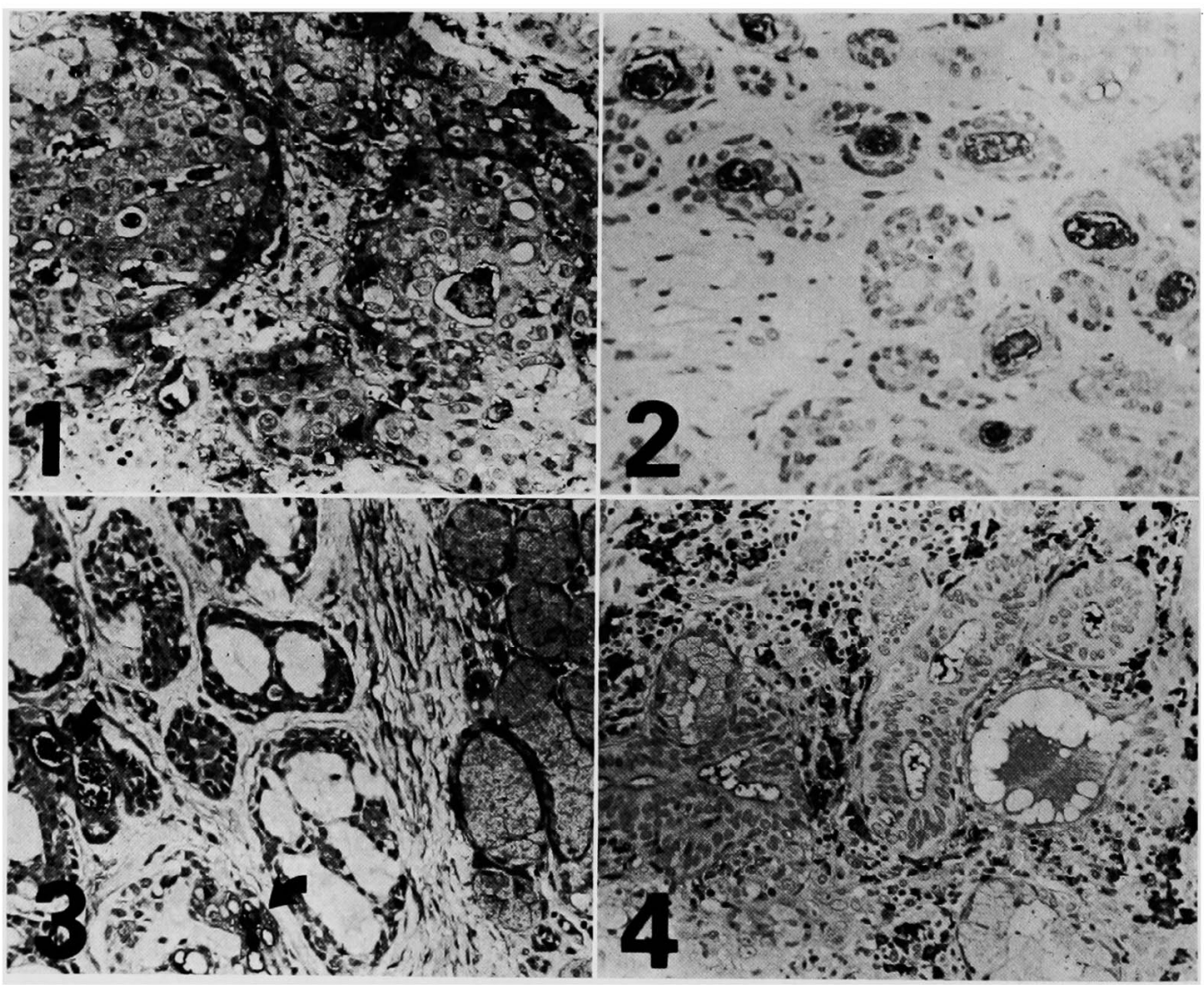

Fig. 1 Immunoperoxidase staining of CEA in mucoepidermoid tumor. CEA was present in small tubules. (Counterstain hematoxylin $\times 250$ )

Fig. 2 Immunoperoxidase staining of CEA in adenoid cystic carcinoma. CEA was present in cytoplasm at the apical cell border and in the lumen of the microcyst in cribriform pattern. (Counterstain hematoxylin $\times 200$ )

Fig. 3 Immunoperoxidase staining of amylase in adenoid cysitc carcinoma. Intense staining of secretory material (arrows) in cribriform pattern and apparently non-neoplastic acini at the periphery of tumor. (Counterstain hematoxylin $\times 200$ )

Fig. 4 Immunoperoxidase staining of $\operatorname{IgA}$ in mucoepidermoid tumor. IgA positive cells were present diffusely in stroma, particularly adjacent to neoplastic duct. (Counterstain hematoxylin $\times 200$ ) 
$\mathrm{kam}^{6)}$

\section{AMYLASE}

The localization of amylase in the neoplastic tissue corresponded to that of CEA, but it is interesting to note that whereas the normal minor salivary glands reacted positively to amylase (Fig. 3), it was negative to CEA.

\section{$\operatorname{IgA}$ and $\mathrm{SC}$}

IgA-producing immunocytes were diffusely present in the stroma, particularly adjacent to the neoplastic duct in PMA and MET (Fig. 4) and generally widely showed a more positive reaction to SC than IgA. Aside from ACC positive for hyalinized stroma in the cribrifirm pattern, all other tumor cells showed negative ACC.

\section{LYSOZYME}

Tumor cells in the neoplastic tissue did not show a clear reaction to positive lysozyme and in PMA had weakly positive results similar to IgA and SC. IgA and SC were all found in the same locality as ACC. Negative lysozyme in the neoplastic tissue confirmed the report by Caselitz ${ }^{7}$.

From our preliminary examination, it was found that CEA and amylase were different from IgA, SC and lysozyme in distribution depending on the malignancy of the tumor. It is interesting to note that amylase was found even when affected by tumor. Further accumulation of case data and analysis will be necessary in order to prove the interrelationships between the above 5 secretory proteins in salivary gland tumors ${ }^{8)}$.

\section{Acknowledgement}

The authors thanks Associate Prof. Dr. F. Ide and Instructor, Dr. T. Iwase for invaluable immunological reagents and advice, and Dr. N. Horie for technical assistance.

Note: While this paper was in press, Caselitz et al. cbserved tumor antigens in parotid neoplasms. (Caselitz, J. et al.: J. Oral. Pathol. 11: 374-386, 1982)

\section{References}

1) Gold, P. and Freedman, S. O.: Demonstration of tumor-specific antigen in human carcinomata by immunological tolerance and absorption techniques. J. Exp. Med. 121: 43.9462, 1965.

2) Rognun, T., Brandteag, P., Orjasater, H. and Fausa, O.: Immunohistochemistry of epithelial cell markers in normal and pathological colon mucosa. Histochemistry 67: 7-21, 1980.

3) Sternberger et al.: The unlabeled antibody enzyme method of immunohistochemistry. $J$. Histochem. Cytochem. 18: 315-333, 1970.

4) Mepham, B., Frater, W. and Mitchell, B. S.: The use of proteolytic enzymes to improve immuncglobulin staining by the PAP technique. Histochem. J. 11: 343-357, 1979.

5) Healey, W. V., Perzin, K. H. and Smith, L.: Mucoepidermoid carcinoma of salivary gland origin. Cancer 26: 368-388, 1970.

6) Ejeckam et al.: Immunohistopathologic study on CEA-like material and immunoglobulin A in gastric malignancies. Cancer 44: 16061614, 1979.

7) Caselitz, J., Jaup, T. and Seifert, G.: Lactoferrin and lysozyme in carcinomas of parotid gland. Virchow. Arch. (Pathol. Anat.) 394: 61-73, 1981.

8) Saito, I. et al.: Immunohistochemical characterization of functional markers in human minor salivary gland tumors. (in preparation) 